Revue internationale P.M.E.

Économie et gestion de la petite et moyenne entreprise

\title{
Vers une théorie financière adaptée aux PME. Réflexion sur une science en genèse
}

\section{Vincent Colot et Pierre A. Michel}

Volume 9, numéro 1, 1996

URI : https://id.erudit.org/iderudit/1008258ar

DOI : https://doi.org/10.7202/1008258ar

Aller au sommaire du numéro

\section{Éditeur(s)}

Presses de l'Université du Québec

ISSN

0776-5436 (imprimé)

1918-9699 (numérique)

Découvrir la revue

\section{Citer cet article}

Colot, V. \& Michel, P. A. (1996). Vers une théorie financière adaptée aux PME. Réflexion sur une science en genèse. Revue internationale P.M.E., 9(1), 143-166. https://doi.org/10.7202/1008258ar

\section{Résumé de l'article}

Ces dernières années, la recherche universitaire en finance s'est penchée avec un succès moyen sur la petite et moyenne entreprise (PME). Dans cet article consacré à la finance entrepreneuriale, nous remontons les courants de la pensée scientifique qui ont modelé les connaissances financières pour éventuellement y déceler des éléments qui ne seraient pas pertinents à l'objet de notre étude et, partant, pour réorienter les efforts de recherche tant théorique qu'empirique.

Élaborée en prenant la grande entreprise comme modèle de référence, la théorie financière moderne, dans une forme pure ou amendée, s'avère souvent impuissante à prendre en compte les caractéristiques managériales de la PME. Nous illustrons ici la faible portée analytique des paradigmes ou méthodologies traditionnellement en vigueur. La théorie de la firme, du fait de son rôle particulier en relation avec la science économique, représente selon nous la pierre angulaire de toute avancée significative en modélisation de la PME: en particulier, les notions de compétence et d'expérience de l'entrepreneur actif sur des marchés en situation de déséquilibre constant doivent prendre le pas sur l'hypothèse non réaliste d'un comportement rationnel basé sur la maximisation des bénéfices à l'équilibre. Enfin, dans un ultime élargissement de notre champ d'investigation, nous analysons le bien-fondé d'une étude purement financière de la PME et les espoirs dont sont porteuses des disciplines scientifiques peu conventionnelles en sciences de gestion.

Notre contribution se veut avant tout révélatrice puis intégratrice de la hiérarchie naturelle des concepts sur lesquels repose le développement de la finance entrepreneuriale.
Ce document est protégé par la loi sur le droit d'auteur. L'utilisation des services d'Érudit (y compris la reproduction) est assujettie à sa politique d'utilisation que vous pouvez consulter en ligne.

https://apropos.erudit.org/fr/usagers/politique-dutilisation/ 


\title{
Vers une théorie financière adaptée aux PME. Réflexion sur une science en genèse ${ }^{1}$
}

Vincent COLOT

Pierre A. MICHEL

Université de Liège, Belgique

\section{MOTS CLÉS}

\section{Recherche universitaire en finance-Finance entrepreneuriale Théorie financière moderne - Théorie de la firme Modélisation de la PME - Compétence et expérience de l'entrepreneur-Disciplines scientifiques peu conventionnelles en sciences de gestion}

\begin{abstract}
RÉSUMÉ
Ces dernières années, la recherche universitaire en finance s'est penchée avec un succès moyen sur la petite et moyenne entreprise (PME). Dans cet article consacré à la finance entrepreneuriale, nous remontons les courants de la pensée scientifique qui ont modelé les connaissances financières pour éventuellement y déceler des éléments qui ne seraient pas pertinents à l'objet de notre étude et, partant, pour réorienter les efforts de recherche tant théorique qu'empirique.
\end{abstract}

1. Cette étude a été présentée au $1^{\text {er }}$ Congrès international francophone de la PME à Carthage (Tunisie) en octobre 1993 et à la 39 Conférence mondiale du Conseil international de la petite entreprise à Strasbourg (France) en juin 1994. Elle a également été retenue comme l'une des cinq meilleures recherches à la $8^{\mathrm{e}}$ Nordic Conference on Small Business Research à l'Université de Halmstad (Suède) en juin 1994. Cette recherche a bénéficié de l'aide financière de la Communauté française de Belgique, du ministère de l'Éducation, de la Recherche et de la Formation (Action de recherche concertée 90 / 94-141). Les auteurs désirent également remercier Christine Thirion, assistante à l'Université de Liège, $\mathrm{H}$. Landström, professeur à l'Université de Halmstad, ainsi que l'arbitre de la revue, pour leurs encouragements et commentaires pertinents lors de la rédaction de ce travail. Toute inexactitude demeure cependant la seule responsabilité des auteurs. 
Élaborée en prenant la grande entreprise comme modèle de référence, la théorie financière moderne, dans une forme pure ou amendée, s'avère souvent impuissante à prendre en compte les caractéristiques managériales de la PME. Nous illustrons ici la faible portée analytique des paradigmes ou méthodologies traditionnellement en vigueur. La théorie de la firme, du fait de son rôle particulier en relation avec la science économique, représente selon nous la pierre angulaire de toute avancée significative en modélisation de la PME: en particulier, les notions de compétence et d'expérience de l'entrepreneur actif sur des marchés en situation de déséquilibre constant doivent prendre le pas sur l'hypothèse non réaliste d'un comportement rationnel basé sur la maximisation des bénéfices à l'équilibre. Enfin, dans un ultime élargissement de notre champ d'investigation, nous analysons le bien-fondé d'une étude purement financière de la PME et les espoirs dont sont porteuses des disciplines scientifiques peu conventionnelles en sciences de gestion.

Notre contribution se veut avant tout révélatrice puis intégratrice de la hiérarchie naturelle des concepts sur lesquels repose le développement de la finance entrepreneuriale.

\begin{abstract}
During the last years, academic research in finance has studied small and medium-sized enterprises with mixed success. In this article devoted to entrepreneurial finance, we return to the origins of the currents of scientific thought, at the root of financial knowledge, in order to detect eventual inadequacies to the object of our study, and consequently, in order to reorient efforts in theoretical as well as empirical research.

Using large firms as reference, modern financial theory, in pure or amended form, often turns out to be incapable to take into account the managerial specificities of small and medium-sized enterprises. We illustrate in this article the limited analytical range of application of the paradigms or methodologies which are traditionally used. According to us, the theory of the firm, because of its particular role in relation to economic science, represents the corner-stone of any significant advance in the application of models to small and medium-sized enterprises. In particular, the notions of competence and experience of the entrepreneur active on markets characterized by a constant disequilibrium ought to replace the less realistic hypotheses of rational behavior based on the maximization of profits at equilibrium. Finally, in an ultimate enlargement of our field of investigation, we analyze the credibility of an exclusively financial study of small and medium-sized enterprises and the possible contribution of some scientific disciplines rarely used in management science.
\end{abstract}

Our study is intended above all to reveal, but also to integrate, the natural hierarchy of the concepts which are at the basis of the development of entrepreneurial finance. 


\section{RESUMEN}

Durante estos ultimos anos, la investigacion academica en finanzas estudio con un exito mitigado la pequeña y media empresa (PyME). En este articulo dedicado a finanzas de las empresas, volvemos a las corrientes del pensamiento cientifico que han modelado los conocimientos en finanzas para descubrir eventuales inadecuaciones al objeto de nuestro estudio y, de alli, reorientar los esfuerzos de investigacion tanto teorica como empirica.

Elaborada tomando a la gran empresa como modelo de referencia, la teoria financiera moderna, en una forma pura o corrigida, se revela muchas veces impotente a tomar en cuenta las especificidades de los directivos de la PyME. llustramos aqui el menguado alcance analitico de los paradigmas o metodologias tradicionalmente en vigor. La teoria de empresa, por su papel particular en relacion con la ciencia economica, representa segun nosotros la piedra angular de todo avance significativo en modelizacion de la PyME: en particular, las nociones de competencia y de experienca del empresario activo en los mercados en situacion de desequilibrio constante tienen que prevalecer sobre la hypotesis no realista de un comportamiento racional basado sobre la maximizacion de los beneficios al equilibrio. Para acabar, en una ultima ampliacion de nuestro campo de investigacion, analizamos el bien fundado de un estudio puramente financiero de la PyME y las esperanzas que entranan las disciplinas cientificas poco convencionales en ciencias de gestion.

Nuestra contribucion quiere ser ante todo reveladora y despues integradora de la jerarquia natural de los conceptos situados a la base del desarrollo de la finanza de las empresas.

\section{LES AUTEURS}

Vincent Colot, licencié en administration des affaires de l'Université de Liège, est chercheur au Service d'analyse financière de cette université.

Pierre A. Michel détient un MBA et un Ph.D. (finance) de l'Université de New York (NYU), il est titulaire de la Chaire d'analyse financière à la Faculté d'économie, de gestion et de sciences sociales de l'Université de Liège, où il occupe la fonction de président de l'École d'administration des affaires. II a également fondé le Centre PME de l'Université de Liège. Son enseignement et ses recherches portent sur la comptabilité des coûts, la théorie financière, la gestion de portefeuille et la finance d'entreprise.

Adresse : École d'administration des affaires, Université de Liège, 7, boul. du Rectorat, B. 31, 4000 Liège, Belgique. 


\section{Introduction}

If any discipline is to be propelled from its origins to a more advanced state, the initial myriad of seemingly incoherent theories, assumptions, notions, rules of thumb, and wives' tales must be organized into a unifying framework for investigation.

M.R. Perryman (1982), p. 378.

Depuis plusieurs années, les responsables politiques et économiques soulignent régulièrement l'importance de la petite ou moyenne entreprise (PME) au sein des pays industrialisés et en voie de développement. Cette préoccupation se renforce aujourd'hui encore. En effet, la crise économique à connotation structurelle qui touche actuellement les pays industrialisés exige une adaptation radicale des agents économiques en termes de flexibilité et de décentralisation. À cet égard, la promotion de la PME en tant qu'ultime vecteur d'innovation et d'emploi s'avère cruciale. Parallèlement, les pays émergeant de régimes dictatoriaux (pays en voie de développement, pays de l'ex-bloc de l'Est) trouvent dans le phénomène d'entrepreneuriat un outil primordial de démocratisation économique et de responsabilisation civile.

Quant à l'engouement scientifique suscité par l'entreprise de taille réduite, il est assez récent. À l'origine de cet intérêt se trouve la prise de conscience de la spécificité de la PME sous beaucoup d'aspects managériaux. Ainsi, ce n'est que depuis peu qu'elle n'est plus considérée comme la réplique miniature d'une grande entreprise. La multiplication des publications, congrès et programmes doctoraux qui lui sont consacrés témoignent suffisamment de ce changement de mentalité.

Toutefois, de graves carences théoriques caractérisent encore la recherche dans le domaine des PME. À vrai dire, il n'existe aucune base théorique reconnue qui rende compte du phénomène d'entrepreneuriat. Cet état de fait constitue un frein au développement de la recherche scientifique, ainsi que le regrettent Bygrave et Hofer (1991). De même, la recherche en finance et PME n'en est qu'à ses balbutiements de formalisation (Ang, 1992). Petty (1991) déplore la faiblesse qualitative quasi généralisée de la recherche dans ce domaine, tandis que Landström (1992), s'il reconnaît l'existence d'études financières empiriques, déplore le peu d'ardeur scientifique à en réunir les résultats au sein d'un cadre théorique globalisateur. Enfin, le ton catégorique de Ang (1992, p. 185) dénote l'ampleur et l'urgence de la tâche à accomplir: «Je puis vous assurer, à ce moment-ci, qu'il n'existe pas de théorie financière unique qui puisse expliquer les comportements des différents types de PME.» 


\section{Perspective}

Une partie importante de la recherche en PME s'est focalisée sur la détermination des traits de personnalité des entrepreneurs : ainsi, selon les études, un entrepreneur est plus ou moins prudent, plus ou moins prédisposé au stress. Même si ces approches psychologiques continuent à inspirer certains chercheurs, un nouveau type d'analyse s'impose de plus en plus ${ }^{2}$. En effet, il y aurait quasiment autant de types d'entrepreneurs que d'entreprises, cela rendant toute formalisation délicate. Selon Gartner (1988), importent principalement les activités mises en œuvre par l'entrepreneur dans la gestion de son entreprise. Les approches psychologiques cèdent le pas aux approches comportementales. Ce point de vue est partagé par d'autres spécialistes de renom comme Ivancevich (1991), Bygrave et Hofer (1991). Ces derniers, qui s'appuient sur la notion de processus entrepreneurial, ont parfaitement synthétisé la nouvelle philosophie de la recherche en PME. Le mécanisme de perception des occasions d'affaires et l'accomplissement des tâches proprement entrepreneuriales constituent dorénavant les pôles incontournables de tout développement scientifique sérieux.

Nous nous mettons ici dans la peau du chercheur en gestion qui, délaissant le «monde rassurant» de la grande entreprise, décide de relever le défi de la finance entrepreneuriale. Notre objectif est de simuler le cheminement conceptuel logique entrepris par ce chercheur confronté à cette nouvelle tâche scientifique. Au fur et à mesure de notre réflexion, nous remontons par paliers, dans «une optique PME», les courants de la pensée scientifique qui ont modelé les connaissances financières actuelles. Nous parcourons ainsi successivement cinq champs d'investigation: 1) la théorie financière moderne, 2) la prise en compte de la réalité des PME, 3) la science économique néoclassique et la théorie de la firme, 4) la gestion : sciences unidisciplinaires ou sciences multidisciplinaires, 5) la conception traditionnelle de la pensée scientifique. Nous ne désirons pas établir une revue exhaustive de la littérature existante. Nous ne prétendons pas élaborer ex nihilo une théorie financière adaptée aux PME. Notre apport se veut avant tout révélateur puis intégrateur de la hiérarchie naturelle des concepts fondant le développement de la finance entrepreneuriale.

Tout en identifiant les carences de chaque approche isolée, nous menons une action curative en deux temps. Dans une première phase, nous remontons méthodiquement chaque palier (maillon de la chaîne) constitutif d'un niveau de connaissance dans le sens hiérarchique inverse au développement de la

2. Le lecteur intéressé par cette question se reportera utilement à l'ouvrage The State of the Art of Entrepreneurship, Sexton et Kasarda (1992). 
finance d'entreprise: ainsi, il nous est loisible de constater si nous pouvons résoudre les problèmes rencontrés au niveau précédent en cernant la «source du mal ». Par cette démarche, nous allons nécessairement à contre-courant de bon nombre d'idées reçues, soulevant par là même d'autres questions, ce qui nous pousse sans cesse à élargir le champ de l'analyse à la recherche de solutions de rechange.

Une fois effectué ce survol exhaustif des domaines à considérer, nous déterminons, après diagnostic, celui ou ceux qui, selon nous, doivent faire l'objet de développements prioritaires. C'est sur le ou les maillons faibles de la chaîne que devront se concentrer les recherches, à la lumière des apports des autres maillons. C'est alors seulement que nous pourrons envisager plus sereinement l'avenir de la recherche en théorie financière adaptée aux $\mathrm{PME}^{3}$. Ce parcours initiatique vers la source du savoir s'apparente à celui du saumon remontant les cours d'eau pour procréer une génération nouvelle au lieu même de sa naissance ${ }^{4}$.

\section{Théorie financière moderne}

Markowitz, Sharpe, Miller, Modigliani, Ross, Roll, Jensen, Meckling, Fama, etc., autant de noms illustres associés au développement de la théorie financière depuis la fin des années 1950. Le chercheur en finance, habitué à côtoyer les modèles théoriques de ces auteurs, a naturellement tendance, dans un premier temps, à les appliquer ex abrupto au domaine des PME.

Une remarque initiale s'impose: évitons de faire de ces acquis théoriques une panacée. En effet, cette science financière est une discipline relativement jeune (comparé à la physique...) qui est encore loin d'avoir répondu à toutes les attentes. Ainsi, Brealey et Myers (1991) synthétisent en fin de leur ouvrage dix problèmes de finance-et non des moindres-encore non résolus. Citons, par exemple, les mystères entourant la structure financière des entreprises ou la controverse autour de la signification des dividendes.

3. Cet article a été rédigé en 1993. Entre-temps, la recherche a progressé. Nous espérons cependant que le lecteur intéressé par ces questions trouvera ici une source d'inspiration originale, parfois volontairement provocatrice, susceptible d'enrichir sa propre réflexion.

4. L'idée de base de cette réflexion nous est venue en écoutant une interview télévisée d'I. Prigogine, Prix Nobel de chimie (1977). Adolescent, il voulait devenir avocat. Plutôt que de choisir automatiquement des études de droit, il comprit que la psychologie lui serait indispensable pour saisir la mentalité des meurtriers. En outre, pour percer les mystères du cerveau, il fallait des connaissances en biologie. Enfin, la complexité de l'architecture neuronale relevait de la chimie, science qui devint le domaine de prédilection du savant belge. 
Qu'advient-il des grands paradigmes de la finance moderne lorsque mis en relation avec la réalité des PME ? Nous analysons ici trois types de recherche qui conduisent à des résultats plus ou moins satisfaisants.

\subsection{La diversification}

Un manque évident d'esprit critique ou une méconnaissance grave des principes de base régissant la gestion des PME peuvent amener le choix d'un modèle inadapté. Dukes, Bowlin et Ma (1992) reconnaissent que le modèle d'évaluation des entreprises de Gordon et Shapiro se révèle incohérent dans le cas de la PME, car il est basé sur l'actualisation de dividendes que peu de PME distribuent régulièrement. Freear et Wetzel (1992) remettent en cause, quant à eux, le principe de diversification inhérent à la gestion de fonds de placement dès qu'il est question de capital de risques à destination de PME. Ce principe de prudence bien connu des spécialistes financiers correspond en essence à la maxime populaire préconisant de ne pas mettre tous ses œufs dans le même panier. Les deux auteurs précités soulignent que l'octroi de capital de risques à des PME s'accompagne le plus souvent de clauses contractuelles de «conseil»: l'investisseur conseille et guide l'entrepreneur afin de renforcer la rentabilité. Cet effort consenti par l'investisseur exige de lui qu'il se spécialise sur les plans technologique et géographique afin de mieux maîtriser tous les aspects de la gestion. Restreindre les risques en matière d'investissement entrepreneurial revient à ne pas diversifier son placement sur plusieurs secteurs d'activité (nationaux ou internationaux), mais bien à faire bénéficier les PME d'une expérience aiguë acquise au sein d'un domaine bien précis.

\subsection{Le CAPM}

Les comparaisons financières entre grandes et petites firmes constituent un pan important de la recherche. Dans ce cadre, un modèle peut s'avérer révélateur de différences sans toutefois en fournir une explication valable. Sharpe (1964) a élaboré un modèle d'évaluation des actifs financiers à l'équilibre (Capital Asset Pricing Model ou CAPM) qui met en évidence une relation linéaire positive entre le rendement espéré d'un actif financier et son risque. Ce risque systématique ou risque de marché est celui que l'investisseur ne peut éliminer et pour lequel il exige donc une compensation en termes de rendement attendu. Ce risque correspond à la sensibilité des rendements de l'actif à ceux du marché. Banz (1981) a découvert que les petites entreprises, à risque (bêta) égal, génèrent-en moyenne-des rendements plus élevés que les grandes entreprises. Depuis lors, de nombreuses études essayent d'expliquer cet effet de taille. Il semble aujourd'hui que l'hypothèse d'inefficience du marché, liée par exemple à des coûts de transaction importants, ne puisse plus être retenue 
comme explication principale. Le CAPM, dans sa conception première, n'est pas capable d'intégrer cet effet de taille. Cette déficience conceptuelle relève d'une explication de nature économique plus que statistique. Cela signifie que le coefficient bêta ne renferme pas, per se, les facteurs explicatifs de l'effet de taille, ainsi que le souligne Jegadeesh (1992). Ce dernier plaide pour une analyse en profondeur des facteurs de risque idiosyncratique (lié aux caractéristiques propres de la PME). Chan et Chen (1991), quant à eux, définissent la notion de firme marginale: du fait de facteurs tels qu'un appareil de production vieillissant ou un endettement excessif, cette firme s'avère plus sensible que les autres aux turbulences de l'environnement économique. Selon ces auteurs, une majorité de petites firmes tendent à présenter cette caractéristique. La taille serait alors secondaire, un effet de marginalité s'imposant comme centre de l'analyse. Dans ce contexte, les auteurs épinglent l'inadaptation du CAPM: "Comme résultat $[\ldots]$ les petites entreprises tendent à être plus risquées que les grandes, et leur risque ne peut vraisemblablement être correctement apprécié par un indice de marché composé majoritairement de titres de grandes sociétés » (p. 1483).

\subsection{La théorie de l'agence}

Certains modèles financiers, qui furent développés pour la grande entreprise, révèlent un potentiel explicatif plus important pour traiter la problématique des PME que pour traiter celle des grandes entreprises. C'est le cas de la théorie de l'agence. Rarement cadre conceptuel a été porteur d'un aussi grand espoir de formalisation financière des PME que ce modèle élaboré par Jensen et Meckling (1976). Cette théorie, basée sur l'existence de conflits d'intérêts, s'inspire de développements antérieurs couvrant les phénomènes de risque moral et d'asymétrie d'informations dont Adam, Farber et Michel (1989) donnent quelques illustrations appliquées aux PME. Le banquier ou le fournisseur de capital à risque (le principal) contrôlent difficilement les agissements du dirigeant de la petite firme (l'agent), surtout par manque de transparence des moyens d'information (pas de marché boursier des entreprises non cotées, la valeur des dirigeants de PME n'est guère sanctionnée sur le marché du travail, etc.). Cette situation particulière entraîne naturellement la croissance des coûts d'agence. Déjà exposée par Pettit et Singer (1985) dans leur important article, la théorie de l'agence demeure défendue par de nombreux auteurs tels que Ang (1991; 1992), Brophy et Shulman (1992).

Toutefois, les premières études empiriques fragmentaires réalisées conformément aux préceptes de cette théorie appellent à la circonspection. Si Suret et Gagnon (1990) ont dégagé certains résultats intéressants, Norton (1991), après son enquête sur des PME à croissance rapide, rejette le bienfondé de la théorie de l'agence à leur égard. Un même constat d'échec découle 
de l'étude de Landström (1992) sur les relations entre entrepreneurs et investisseurs privés en Suède. Il observe, entre autres, que la fréquence des contacts entre les deux parties et l'ampleur effective de leur coopération ne sont pas déterminées principalement par le niveau de contrôle requis par les investisseurs. Une autre différence par rapport aux prescriptions de la théorie de l'agence, c'est qu'aucun lien n'est décelé entre la participation de l'entrepreneur dans le capital de sa firme et le comportement de «surveillance» (monitoring behaviour) de l'investisseur privé.

Comment expliquer les conclusions de ces tests empiriques? Si la philosophie générale de la théorie de l'agence est plaisante, certaines hypothèses en amont du modèle semblent non appropriées au contexte de la PME. Nous reviendrons sur ce point à un stade ultérieur de notre réflexion.

\section{Prise en compte de la réalité des PME}

La plupart des études théoriques et empiriques portant sur les PME et conduites conformément à l'orthodoxie en vigueur ont abouti à des résultats moyens ${ }^{5}$. Conséquemment à ces désillusions, d'autres chercheurs ont été tentés d'ajouter une «coloration» PME aux modèles et outils existants. Dans un souci de confort intellectuel, ils ont voulu retrouver une cohérence au sein de la théorie financière. Cependant, loin de constituer l'aboutissement des efforts de recherche, cette tendance n'occupe-selon nous-que le second palier du processus de réflexion méthodologique qui devrait nous conduire à l'élaboration d'une science financière adaptée aux PME.

\subsection{Adaptation des paradigmes existants}

Quelques chercheurs s'attellent à retravailler des modèles ou paradigmes en vigueur afin de les adapter à une «logique $\mathrm{PME}$ ». Ces techniques d'adaptation ne sont pas exemptes de critiques. À titre d'exemple, nous montrons successivement ci-après, d'une part, les avatars d'une relation d'indépendance entre deux variables avancées, en 1958, par Modigliani et Miller et, d'autre part, une tentative visant à compléter une équation fondamentale, celle du CAPM.

Modigliani et Miller (1958) ont conclu, sous certaines hypothèses (absence de taxes, etc.), au constat d'indépendance entre la valeur d'un investissement et la nature de son financement. Cette relation est actuellement contestée sous l'éclairage particulier de la PME et de son environnement. Une des hypothèses centrales du modèle concerne l'existence de marchés financiers

5. Voir l'ouvrage de McMahon, Holmes, Hutchinson et Forsaith (1993). 
en concurrence parfaite. Si une grande entreprise a un accès relativement aisé à des capitaux de types diversifiés, il n'en va pas de même de la PME dont la marge de manœuvre est nettement plus réduite et qui doit encourir des coûts parfois importants lorsqu'un financement externe s'avère nécessaire. Plusieurs auteurs se sont penchés sur la proposition de Modigliani et Miller de différentes manières. Ils ont cherché à établir, pour les PME, une relation de causalité entre les deux mêmes facteurs de financement et d'investissement. Klinge (1992) s'est intéressé aux ressources d'autofinancement dégagées par les entreprises: son analyse empirique concerne la dépendance potentielle des décisions d'investissement à la présence de capacités d'autofinancement. Les résultats dégagés ne sont que peu significatifs. Le modèle de Berkovitch et Narayanan (1993) envisage la causalité inverse. Partant de l'hypothèse de nullité des bénéfices mis en réserve, ces auteurs prennent en compte les perspectives de profitabilité de tout projet d'investissement et les conditions économiques prévalant lors de la décision d'investir: ces deux critères exercent, selon eux, une influence sur le type de financement adopté. Les auteurs recommandent les PME comme étant la population idéale pour tester empiriquement leur modèle. Quant à Brophy et Shulman (1993), ils avancent l'idée de relations réciproques entre financement et investissement dans un contexte de développement de l'innovation. Cette conclusion, issue de leurs travaux de modélisation, contredit Modigliani et Miller alors que les hypothèses de départ sur lesquelles les auteurs se sont basés respectaient la littérature en vigueur.

S'inspirer des paradigmes existants est limitatif: on aborde les mêmes problèmes sous une perspective dont le caractère approprié reste à démontrer. De plus, la modification de certaines hypothèses d'un modèle - qui aboutit de ce fait à la non-vérification de celui-ci-ne constitue pas en soi un progrès substantiel. Il convient à tout le moins de se demander si toutes les hypothèses à revoir ont fait l'objet d'un examen scrupuleux.

En outre, en cas de modélisation ultérieure, il est difficile de se démarquer du modèle d'origine, surtout lorsque cela s'avère nécessaire à cause de l'importance ou du nombre d'hypothèses modifiées. Voyons ci-après un exemple plus net de ce type d'aveuglement scientifique. Monahan, Stout et Barenbaum (1992), étudiant l'effet de taille, avancent non sans une certaine audace: "Il est possible [...] que le processus d'évaluation des actions pour les petites entreprises à capital privé diffère (probablement de façon systématique) de celui appliqué aux grandes firmes dont les titres se traitent en bourse» (p. 3).

Dans leur étude, loin de réfuter le CAPM sous-jacent à la découverte du phénomène, ils en ont présenté une formule légèrement modifiée, en y ajoutant un facteur destiné à rendre compte du supplément de risque de marché associé aux petites entreprises. Force est de reconnaître que les altérations apportées 
aux hypothèses de départ sont telles que l'étude entreprise ne cadre plus avec le paradigme de référence.

\subsection{La PME comme point de départ de l'analyse}

Une voie d'analyse intéressante semble être la prise en compte explicite d'une dimension temporelle: en effet, une PME connaît des phases de croissance nettement plus marquées que les grandes entreprises. Ces perspectives de «cycle de vie» de la PME ont déjà débouché sur certaines analyses empiriques et théoriques. Keeley et Turki (1992) ont ainsi analysé l'évolution du risque de petites entreprises au cours des phases de développement successives. Par ailleurs, s'attelant à la structure financière des PME, Shulman, Cooper et Brophy (1992) mettent l'accent sur l'évolution des moyens de financement au fur et à mesure de la croissance de l'entreprise.

Deux commentaires s'imposent à ce stade-ci de notre analyse. Premièrement, Sexton (1988) attire l'attention sur le fait que la croissance n'est pas un phénomène inéluctable ou automatique. La portée des travaux susmentionnés n'est donc pas générale. Deuxièmement, les outils utilisés par ces auteurs relèvent de la panoplie de la finance traditionnelle. Nous avons déjà abordé la question de la théorie de l'agence à laquelle recourent Shulman et al. (1992). Quant à Keeley et Turki (1992), au prix d'hypothèses restrictives, ils ont décelé une analogie: selon eux, dans une firme jeune les investissements répétés (entre les phases de développement) peuvent s'assimiler à un processus d'options composées en vue de mesurer une relation risque-rendement. Outre une dénaturation de la réalité pour faire correspondre celle-ci au modèle désiré, cette approche recèle une autre déficience: elle n'éclaire nullement l'analyse sur les types réels de perception du risque et les moyens mis en œuvre pour réduire ce risque. Cette dernière considération doit, d'après Ruhnka et Young (1991), guider les recherches ultérieures dans « un cadre comportemental plus complet ».

Les critiques relevées à ce palier de connaissance amènent l'interrogation suivante: les PME ne diffèrent-elles des grandes entreprises que sur certains sujets liés à certaines caractéristiques ou bien nécessitent-elles un renouveau intégral des concepts et des techniques en vue d'élaborer une théorie financière qui corresponde à leur réalité ? Dans la section suivante, nous dépassons le référentiel purement financier; nous examinons les courants de pensée au sein desquels ont été façonnés les grands modèles financiers et évaluons leur pertinence au regard de la réalité des PME. Toute remise en cause de ces courants de pensée devrait s'assimiler à une révolution conceptuelle... 


\section{Science économique néoclassique et théorie de la firme}

La théorie financière moderne s'est développée dans le cadre plus large de la science économique néoclassique. Cette dernière s'articule autour de deux notions centrales : la théorie de l'équilibre général et la maximisation des profits comme rationalité des agents économiques.

La théorie de l'équilibre général, dans laquelle s'inscrivent des modèles financiers aussi fondamentaux que le CAPM ou les propositions de Modigliani et Miller, s'appuie sur le concept d'équilibre walrassien (modélisé par Arrow et Debreu). Selon ces modèles, l'existence d'échanges entre acteurs économiques est due à la présence de l'équilibre, ce qui exclut toute possibilité de déséquilibre. D'après De Vroey (1987), une telle hypothèse concorde mieux avec les principes d'une économie planifiée qu'avec ceux d'une véritable économie de marché : il y a conjonction et assimilation des notions de «processus» et d' «existence» de l'équilibre. Kaish et Gilad (1991) analysent les implications extravagantes d'une telle logique: l'équilibre stable des marchés concurrentiels annihile toute opportunité de profit, niant par là-même tout phénomène d'entrepreneuriat. La découverte de niches de développement par de nouvelles firmes nécessite, a contrario, une situation de déséquilibre.

En réponse au caractère non approprié des modèles d'équilibre, des écoles de pensées économiques nouvelles ont vu le jour, telles que l'École autrichienne et le courant évolutionniste schumpétérien. Ces approches, qui convergent sur de nombreux points, reconnaissent les situations de déséquilibre comme principe inhérent du capitalisme et centrent leur analyse sur l'entrepreneur. Ce renouveau de la réflexion a engendré dans la foulée quelques tentatives de redéfinition des contours de la théorie de la firme. Dans sa conception traditionnelle, et toujours dominante, la théorie de la firme de philosophie contractuelle s'inscrit dans une logique économique néoclassique de maximisation des profits à l'équilibre. Les partisans de cette approche nient la pertinence des notions de firme et d'entrepreneur: tout service rendu peut faire l'objet d'un contrat et les relations contractuelles constituent la seule réalité économique soumise à la maximisation. Notons que la théorie de l'agence occupe une fonction de modélisation importante au sein du courant contractuel. Se démarquant de cette conception, Foss (1993) plaide ouvertement pour une théorie de la firme centrée sur la notion de compétence de l'entrepreneur qui, selon lui, ne peut être sujette à contrat :

La compétence est en réalité une vision de la rationalité, ce qui est très différent de la maximisation : les fins et les moyens ne sont pas simplement fournis aux décideurs par un processus historique non vérifié. Souvent, les structures de décisions doivent être élaborées par les agents eux-mêmes. C'est la signification de la notion de compétence que l'on qualifie d'entrepreneuriat. (p. 134) 
Landström (1992), dans son essai infructueux d'appliquer la théorie de l'agence aux PME, incrimine l'hypothèse de maximisation des profits par les entrepreneurs et investisseurs privés comme cause majeure de cet échec.

Dans le même ordre d'idée, l'évaluation du risque liée à l'effet de taille ne peut donc se concevoir selon les termes de Schwert $(1983$, p. 10): «[...] de façon à expliquer correctement "l'effet de taille", une nouvelle théorie doit être développée qui soit cohérente avec le comportement rationnel de maximisation de tous les acteurs.»

La compétence de l'entrepreneur n'est-elle pas au centre de la notion de risque que de nombreux auteurs tentent en vain d'appréhender par les modèles et outils de la finance contemporaine? Nous pensons que des recherches complémentaires doivent être menées dans cette voie en vue d'aboutir à une tentative de modélisation. Gibson (1992), dans l'optique de l'École autrichienne, dresse un portrait de l'entrepreneur qu'il présente comme réaliste. Selon lui, l'entrepreneur n'évalue pas rationnellement les possibilités d'investissements au moyen d'une information financière rigoureuse. L'expérience est le seul guide de ses actions. Cette constatation doit nous conduire, par une meilleure compréhension de la PME, à remodeler nos modèles d'évaluation suivant la logique propre de l'entrepreneur. Ce type d'analyse «révolutionnaire» réduit la portée du dilemme énoncé par Ang (1992, p. 189): «[...] des modèles théoriques normatifs pour la plupart doivent être utilisés pour expliquer les observations empiriques positives. Pour décrire les comportements observés chez les PME, un chercheur doit accepter que ces entreprises sont susceptibles de commettre des erreurs, soit en raison d'une confiance excessive ou simplement de l'ignorance.»

Ces notions de compétence et d'expérience au centre des activités de l'entrepreneur peuvent-elles être appréhendées à travers des préoccupations purement financières? Nous touchons ici pour la première fois au bien-fondé même d'une théorie financière adaptée aux PME, nouvelle étape importante de notre investigation.

\section{Gestion : sciences unidisciplinaires ou sciences multidisciplinaires}

Devant la perplexité des chercheurs, il convient de se demander si le champ d'investigation «finance et $\mathrm{PME}$ » constitue une préoccupation scientifique digne d'intérêt (Petty, 1991). Plus précisément, peut-on isoler la fonction financière des autres grandes fonctions de l'entreprise et principalement dans le cadre d'une entreprise où ces fonctions ne font pas l'objet de services distincts? Ce débat, qui n'est pas neuf, occupe une place centrale dans les développements théoriques de l'entrepreneuriat. 
De nombreux auteurs prennent fermement position pour un renforcement de l'interdisciplinarité au sein des sciences de gestion. La départementalisation poussée à laquelle on assiste dans les universités - et qui est calquée sur le fonctionnement des grandes entreprises - est vue comme un frein au progrès scientifique (Ivancevich, 1991; Macmillan et Katz, 1992). Gibb (1992) va encore plus loin dans sa critique du milieu universitaire dont il dépeint les rivalités internes entre disciplines et facultés.

Cette amertume grandissante à l'égard des disciplines isolées de la gestion appelle deux commentaires. Premièrement, nous soupçonnons cette tendance marquée à l'interdisciplinarité d'être née de l'échec des tentatives d'application mécanique des théories traditionnelles au domaine «PME et entrepreneuriat». À notre avis, le statu quo des visions traditionnelles dans un cadre interdisciplinaire ne résoudra rien. Deuxièmement, une distinction peut utilement s'opérer entre la recherche fondamentale et les besoins des entrepreneurs. Si la recherche en finance peut s'enrichir d'une vue plus globale de la gestion, il n'en demeure pas moins que de nombreuses faillites pourraient être évitées si les entrepreneurs disposaient d'outils financiers simples correspondant à leur rationalité. Leur méfiance à l'égard des activités de formation et de "consultance» nécessite le développement de systèmes experts spécialisés (McMahon, 1990). Dans cette optique, nous sommes d'avis de ne pas encourager la dissolution totale des disciplines managériales dans un magma entrepreneurial indifférencié.

Illustrons l'idée qu'une approche «purement » financière peut intégrer des considérations «étrangères » telles que la stratégie ou l'acquisition d'informations.

Un domaine d'investigation essentiel réside dans les relations entre demandeurs (entrepreneurs) et offreurs de capitaux (banques, investisseurs spécialisés en capital à risque). De nombreuses études ont déjà souligné la difficulté éprouvée par les gestionnaires de PME à se procurer des fonds externes. Il y a un double phénomène de méconnaissance et de rareté des sources de financement destinées aux PME. Pour tenter de remédier à cet état de fait, la recherche en finance doit se fixer comme mission prioritaire d'approfondir l'analyse comportementale des deux types d'agents économiques en présence. Tout éclaircissement sur le processus de prise de décision de l'un profite à l'autre par une meilleure compréhension des intérêts de chacun, ce qui ne peut que faciliter les conditions d'octroi d'un prêt basé sur une nécessaire confiance réciproque. L'objectif est donc d'inspecter deux boîtes noires, sources d'incertitude et donc de suspicion légitime. La démarche est naturellement dynamique : le fournisseur de capitaux modifiera ses critères de décision au fur et à mesure qu'il acquerra une meilleure connaissance du processus entrepreneurial, tandis que l'entrepreneur veillera à certains paramètres de gestion dès qu'il sera conscient de l'importance qu'ils peuvent revêtir pour l'offreur de capitaux 
sollicité. Il s'agit ici de concilier deux rationalités différentes, à savoir, d'une part, les préoccupations sous-optimales de l'entrepreneur centrées sur le marché et, d'autre part, une politique plus optimaliste de l'offreur de capitaux qui doit choisir entre plusieurs projets d'investissement pour allouer un capital limité.

Hall et Hofer (1993) font ressortir l'existence d'un processus d'évaluation par les fournisseurs de capitaux à risque : cette analyse se décompose en plusieurs étapes auxquelles correspondent des critères différents. Au terme d'une enquête sur le terrain, ils remarquent que les aspects financiers et stratégiques à strictement parler ne sont envisagés que dans les phases avancées de la négociation. Le profil financier du candidat entrepreneur est néanmoins appréhendé de façon globale dès le départ par le biais de la nature de l'activité projetée. Le prêteur évalue directement le risque lié au secteur d'activité envisagé et peut, à partir de ce seul critère, rejeter l'idée d'un financement, quelles que soient par ailleurs les qualités intrinsèques du projet. Dans une optique comparative, Rosman et O'Neill (1993) se sont penchés sur l'importance relative des critères utilisés dans l'analyse d'une demande de financement, selon qu'il s'agit de capital à risque ou d'un emprunt classique de type bancaire. Dans l'ensemble, les fournisseurs de capital à risque accordent plus d'intérêt aux considérations d'ordre stratégique, tandis que les banquiers portent principalement leur attention sur les résultats financiers de l'entreprise. Cette tendance fournit en partie la raison pour laquelle l'emprunt bancaire n'intervient pas ou peu dans les premières phases de développement d'une PME, faute d'historique sur la santé financière.

Quoi qu'il en soit, la PME, avec ses caractéristiques propres déjà signalées dans cet article, exige un accroissement sinon un renouvellement des outils d'analyse utilisés habituellement en sciences de gestion. Avant d'établir un diagnostic et d'édicter quelques mesures prescriptives, il faut encore étudier une étape afin de compléter notre tour d'horizon des domaines à considérer en vue d'acquérir une vision exhaustive et objective de la situation de la recherche en finance et PME.

\section{Conception traditionnelle de la pensée scientifique}

De nombreux développements dans la connaissance de la finance d'entreprise sont basés sur des concepts mathématiques. Comme chacun le sait, le choix des techniques n'est pas neutre dans l'élaboration d'un modèle. Ainsi, la régression linéaire (simple ou multiple) - par ailleurs, largement utilisée par les chercheurs en finance - est critiquable lorsqu'il s'agit d'étudier un phénomène entrepreneurial. En effet, cette technique économétrique s'accommode mal de modèles instables soumis à des variations brutales, lesquelles sont des caractéristiques de l'évolution des PME (Bygrave et Hofer, 1991). 
Dès lors, pour traiter efficacement le sujet qui nous intéresse, doit-on élargir les outils scientifiques utilisés en gestion en recourant à des domaines «étrangers», afin de rechercher des analogies à pouvoir explicatif ? La finance a déjà ainsi connu des avancées décisives: rappelons que le célèbre modèle d'évaluation des options de Black et Scholes (1973) a été développé à partir de l'équation de la chaleur utilisée en physique. En cette phase initiale de développement de la recherche en finance entrepreneuriale, et malgré la scission classique entre les branches scientifiques, il relèverait de l'ostracisme le plus mal venu de dédaigner les avancées des autres sciences. Bygrave et Hofer (1991) estiment que tant la théorie des catastrophes (mathématiques) que la théorie des changements d'état (physique) peuvent s'appliquer à l'instabilité caractérisant le processus entrepreneurial. Dans un registre différent, un des principaux problèmes rencontrés dans l'étude des PME est l'aspect fragmentaire et peu fourni des données à analyser. Dans des domaines aussi variés que l'épidémiologie, la paléontologie, l'archéologie, la criminologie ou l'histoire, les scientifiques sont habitués à tirer des conclusions à partir de données minimales.

Macmillan et Katz (1992) considèrent ces sciences comme des sources potentielles d'inspiration pour les chercheurs en gestion des PME. Pour traiter de la probabilité de faillite des PME, ils proposent un cadre conceptuel de référence calqué sur la méthodologie de l'épidémiologie. Ils se basent sur les caractéristiques du financement entrepreneurial en termes de fréquence d'occurrence des opérations de financement et de confidentialité (ou obscurité) des données afférentes. Ils proposent donc une analogie appelée à guider les chercheurs dans l'analyse des problèmes de survie des PME (tableau 1).

À partir de cette typologie, ils recommandent l'adoption d'une démarche en trois étapes : l'observation de cas sur le terrain, la recherche en laboratoire pour développer des hypothèses et des modèles de simulation, et les études de population à connotation statistique. Ces auteurs pensent que la science entrepreneuriale, illustrée ici par un thème financier, ne peut être qu'enrichie par ce type d'apports méthodologiques et théoriques.

Bygrave (1993) se montre critique de ces tentatives de développement qu'il considère comme précipitées :

Il y a beaucoup trop d'éléments manquants pour que l'on puisse développer des modèles scientifiques «souples» avec des théories scientifiques «dures». Il me semble que sans ces liens logiques, une théorie biologique ne devrait jamais être la base d'un modèle d'analyse scientifique du comportement humain. Elle devrait plutôt servir de démonstration métaphorique à l'effet que le système humain, dans certaines circonstances, peut présenter des caractéristiques parfois similaires à celles révélées par des organismes beaucoup plus primitifs. (p. 260) 
TABLEAU 1

Confidentialité et fréquence en épidémiologie et entrepreneuriat

\begin{tabular}{lcc}
\hline $\begin{array}{l}\text { Fréquence } \\
\text { d'occurrence }\end{array}$ & Confidentialité des données \\
\hline Élevée & Cancer & Faible \\
Faible & $\begin{array}{c}\text { (investisseurs en capital à risque } \\
\text { informel « anges d'affaires })\end{array}$ & $\begin{array}{c}\text { Rhume banal } \\
\text { (prêts bancaires) }\end{array}$ \\
& SIDA & Lèpre \\
& («Blanchiment d'argent») & $\begin{array}{c}\text { (investisseurs en capital à risque } \\
\text { institutionnel) }\end{array}$ \\
\hline
\end{tabular}

Source : I.C. Macmillan et J.A. Katz (1992), « Idiosyncratic milieus of entrepreneurial research : the need for comprehensive theories ", Journal of Business Venturing, vol. 7, no 1, p. 4.

L'auteur vise tout particulièrement les applications entrepreneuriales dérivées de l'écologie de la population. Il épingle quelques-unes des limitations actuelles de cette approche que d'aucuns considèrent comme une panacée. Les modèles développés jusqu'à présent ne possèdent pas de pouvoir prédictif fiable; leur nature analytique n'est pas en mesure de rendre compte des discontinuités inhérentes à l'entrepreneuriat. Tout résultat ne peut être analysé que par rapport à une population de firmes considérées comme des boîtes noires : en conséquence, cette théorie n'est déterministe que dans un sens probabiliste vu qu'aucun enseignement ne peut en être dégagé au profit d'une entreprise individuelle. Se penchant sur la théorie du chaos, science de la turbulence par excellence, Bygrave n'est guère plus optimiste quant aux apports à en attendre. Selon lui, les échecs répétés de processus chaotiques appliqués aux populations biologiques ne laissent augurer aucun résultat probant en matière entrepreneuriale.

\section{Diagnostic et prescriptions}

Afin d'apprécier le développement de la recherche en finance entrepreneuriale, nous venons de procéder à une vue d'ensemble «par paliers» du processus scientifique (figure 1).

Cette démarche présente de multiples intérêts. Désormais, nous pouvons mieux cerner les limites des approches partielles reposant souvent sur des hypothèses incorrectes. Parallèlement, nous découvrons le potentiel de nombreuses études lorsque celles-ci s'intègrent dans une analyse dynamique multidimensionnelle. Ce renouveau de la pensée analytique nous permet de mieux évaluer les apports des uns et des autres et d'en mesurer la portée le long de la chaîne épistémologique. De même, nous pouvons plus facilement proposer des ébauches d'explication et de solution aux impasses devant lesquelles les auteurs se trouvent souvent. 


\section{Figure 1}

Élargir l'horizon de la recherche en remontant à l'origine du processus de développement scientifique

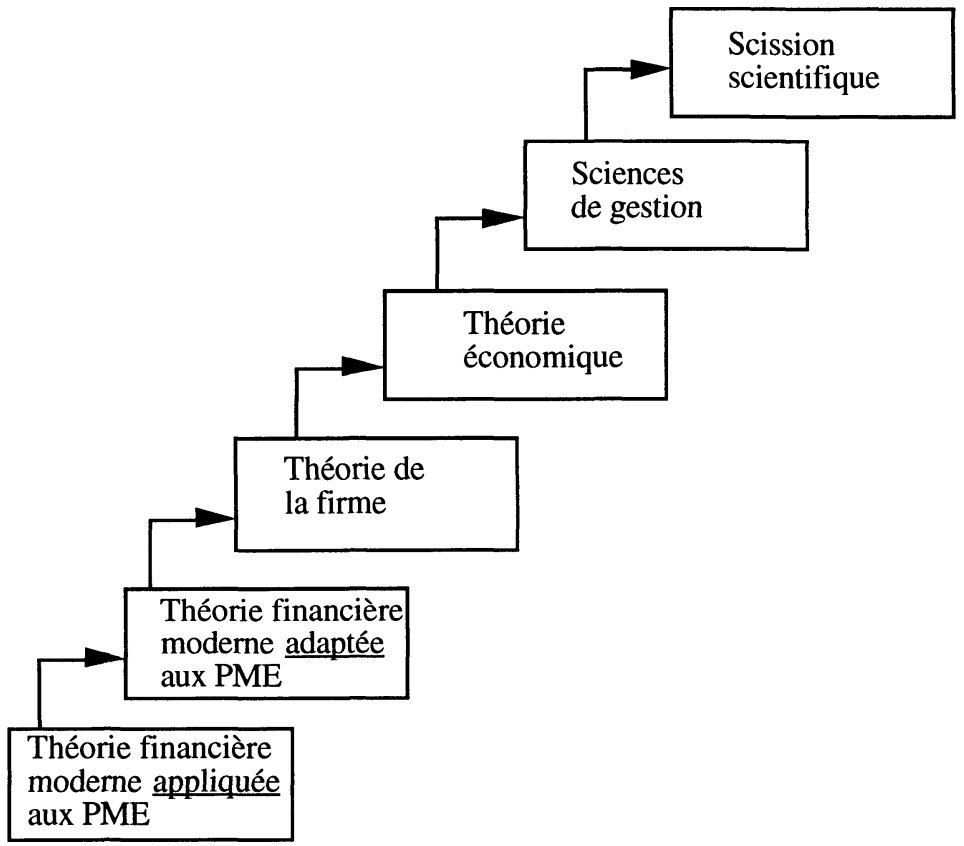

Il est clair que les inquiétudes et doutes de nombreux scientifiques proviennent d'un manque de méthode dans l'analyse. Nous constatons que la majeure partie de la recherche dans le domaine «finance et PME» s'est focalisée sur les deux premiers et sur les deux derniers paliers. L'application et l'adaptation de la théorie financière moderne n'ont pas toujours produit des résultats encourageants. Cependant, de tels développements furent à la fois naturels et nécessaires. En effet, utiliser le matériel théorique existant relevait d'un bon sens apparent et a de ce fait permis, à la lumière des échecs constatés, de mieux orienter les recherches ultérieures. À présent, l'objectif est de dépasser ce stade pour enfin réaliser un progrès normatif. Quant à nos réflexions philosophiques sur le concept des sciences de gestion et leur place parmi les autres sciences, elles ne doivent pas être vues comme des réactions directes aux déficiences de la théorie financière moderne.

La solidité d'une chaîne se mesure à celle de son maillon le plus faible. L'élargissement de l'horizon de notre analyse est à l'origine de la découverte 
de ce maillon, reliquat de la pensée néoclassique (figure 2): il s'agit de la théorie économique et de la théorie de la firme développées à partir du concept de la grande entreprise. Outre cette approche traditionnelle, incompatible avec les caractéristiques des PME, ces théories présentent encore plusieurs points faibles. Elles sont néanmoins cruciales : elles sont la première source d'inspiration de la théorie financière moderne et leur position centrale est, per se, incontournable. Leur fragilité découle de leur importance: le fait qu'elles ne conviennent pas aux PME entraîne la paralysie de tout le processus de développement scientifique. Enfin, les économistes font montre d'une certaine réticence à revoir des modèles à forte complexité mathématique. Prenons garde : nous ne requérons pas un approfondissement isolé et aveugle de ces notions. Cependant, nous considérons ces théories comme le champ d'investigation prioritaire, car elles sont un préalable au développement harmonieux d'une théorie financière réellement adaptée aux PME. Dans cette optique, la réflexion peut se nourrir utilement des résultats dégagés aux deux premiers paliers, et cela, au fur et à mesure que l'on renonce aux hypothèses traditionnelles. En outre, l'évolution des pensées scientifique et managériale (deux derniers paliers) semble fournir des voies de recherche nouvelles, aptes à donner plus de réalisme à toute modélisation potentielle.

La figure 3 est la synthèse de notre méthodologie en termes de modélisation théorique. Lorsque les chercheurs auront une meilleure compréhension des conflits de rationalité entre les acteurs économiques sur des marchés en perpétuel déséquilibre, ils envisageront plus sereinement les différents aspects du financement et de l'investissement propres aux PME. C'est pourquoi, et à condition de s'inscrire dans ce cadre de réflexion rénové, toute avancée en sciences de gestion ou toute application fructueuse d'une autre science seront des atouts importants pour atteindre l'objectif de mise au point d'une théorie financière adaptée aux PME.

Si nous repoussons davantage l'horizon de notre recherche, deux autres questions méritent une prise de position. Premièrement, nous pouvons étudier l'existence éventuelle et la nature des relations potentielles entre la théorie financière appliquée aux grandes entreprises et celle pour les PME. Deuxièmement, est-il envisageable d'assister un jour à l'émergence d'une théorie financière globale, comparable à la théorie universelle (theory of everything) recherchée par les physiciens?

Un des premiers apports de l'intérêt porté aux PME fut la prise de conscience progressive des limites de la théorie financière moderne. Au-delà des questions fondamentales qu'elle a laissées sans réponse, elle ne peut être appliquée aveuglément à tout type d'entreprise. Cette désacralisation des réalisations du courant néoclassique a ouvert, à notre avis, une brèche qui devrait 
FIGURE 2

Localiser le maillon faible

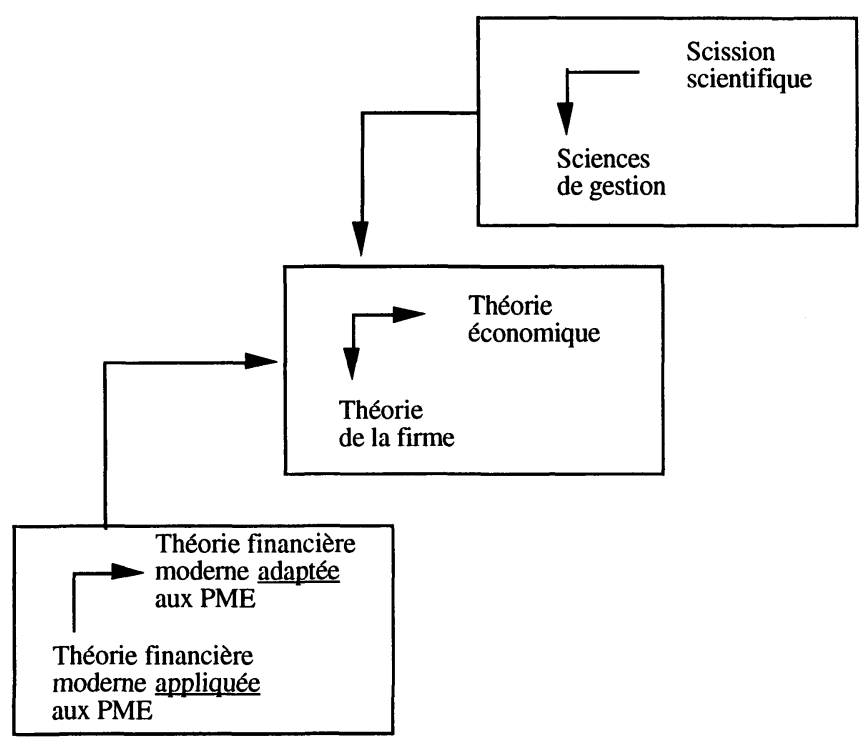

FIGURE 3

Finaliser la modélisation

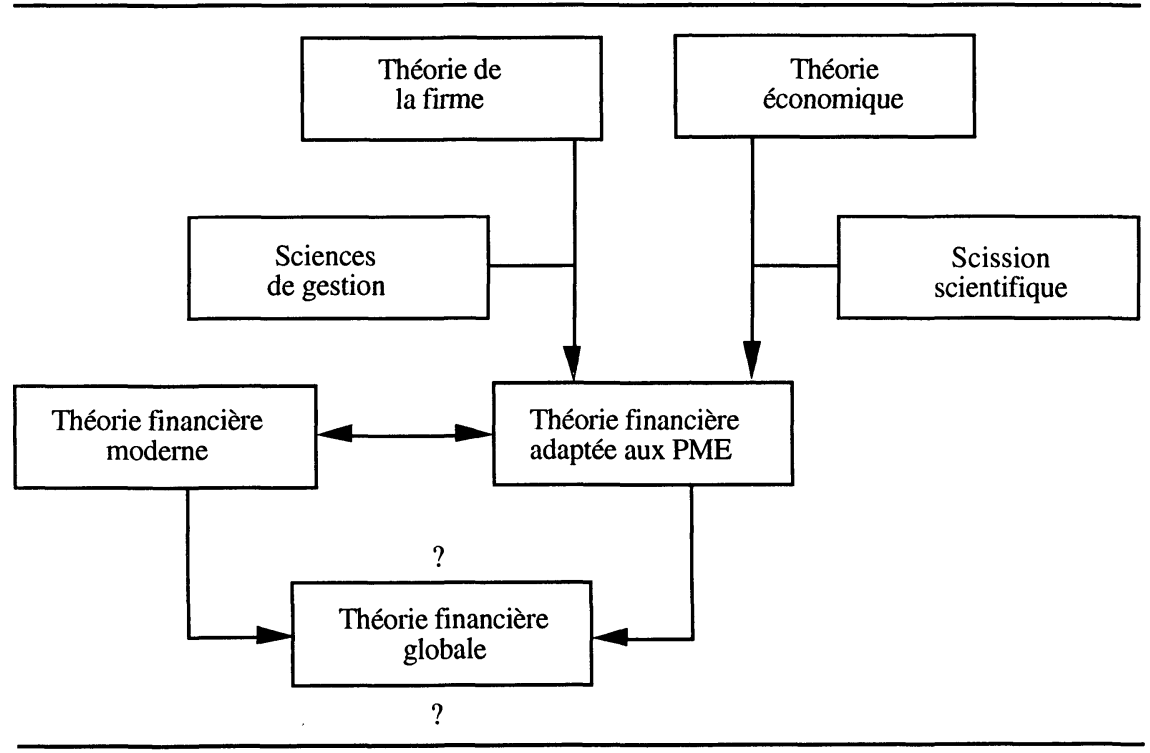


mener à une refonte fondamentale des connaissances en science financière. Des développements similaires à ceux constatés en entrepreneuriat s'observent également en finance "classique» dans le but d'élaborer une théorie plus avancée sur les plans explicatif et prescriptif de la réalité. De même, dans le domaine des marchés boursiers, on trouve des études qui mettent l'accent sur le profil psychologique des investisseurs ou qui rejettent l'hypothèse d'efficience au profit d'un processus chaotique de fixation des prix. Quant à Myers (1990), il avance l'idée d'une théorie organisationnelle de la structure du capital. Ces recherches tentent d'intégrer les deux branches de la finance d'entreprise dans une même volonté d'enrichir et de diversifier l'analyse. Cette tendance n'est cependant pas encore suffisante par elle-même pour envisager une théorie financière globale.

Deux autres facteurs, dont l'évolution reste incertaine, peuvent se conjuguer pour favoriser la réalisation d'une fusion théorique. D'une part, la concurrence de plus en plus âpre touche davantage les grandes entreprises qui, par réaction, recourent à des structures flexibles de production et de gestion. C'est la raison pour laquelle les politiques de décentralisation par des unités plus petites se multiplient. Ce phénomène connu sous la dénomination d' «intrapreneurship » vise à favoriser l'initiative à des échelons de réaction et de décision plus proches du marché. D'autre part, à mesure que progressera la recherche en finance entrepreneuriale, les dirigeants de PME maîtriseront mieux les aspects financiers de leur entreprise et auront plus tendance à les intégrer dans leurs décisions. C'est pourquoi les chercheurs doivent développer des outils et des modèles réellement opérationnels pour les PME. Cette double évolution pourrait conduire à un rapprochement partiel de l'environnement et des comportements des dirigeants de toutes les entreprises, quelle que soit leur taille.

\section{Conclusion}

La finance «PME et entrepreneuriat» constitue une discipline encore en genèse. Devant le bouillonnement théorique et empirique actuel, il nous a paru opportun d'ordonner quelque peu la réflexion. Partis de la théorie financière existante, nous sommes remontés jusqu'à la critique de la scission traditionnelle entre branches scientifiques. Nous espérons que cette méthodologie d'analyse permettra au lecteur de mieux comprendre les apports des uns et des autres et favorisera l'éclosion de nouvelles approches. Quelques chercheurs en finance entrepreneuriale ont déjà ébranlé un certain nombre de croyances.

En effet, la théorie financière traditionnelle, basée sur des hypothèses trop éloignées de la réalité des PME, ne peut servir utilement de référence. La pierre angulaire de la recherche demeure les progrès à réaliser dans les domaines de la science économique et de la théorie de la firme. 


\section{Bibliographie}

ADAM, M.C., A. FARBER et P. Michel (1989), « Théorie financière et PME », Nouvelles de la Science et des Technologies, vol. 7, $\mathrm{n}^{\circ} 3$, p. 59-64.

ANG, J.S. (1991), "Small business uniqueness and the theory of financial management », Journal of Small Business Finance, vol. 1, nº 1, p. 1-13.

ANG, J.S. (1992), «On the theory of finance for privately held firms », Journal of Small Business Finance, vol. 1, no 3, p. 185-203.

BANZ, R.W. (1981), «The relationship between return and market value of common stocks », Journal of Financial Economics, vol. 9, no 1, p. 3-18.

Berkovitch, E. et M.P. NARAyANAN (1993), « Timing of investment and financing decisions in imperfectly competitive financial markets », Journal of Business, vol. $66, \mathrm{n}^{\circ} 2$, p. $219-248$.

BLACK, F. et M. SCHOLES (1973), «The pricing of options and corporate liabilities », Journal of Political Economy, vol. 81, $\mathrm{n}^{\circ} 3$, p. 637-654.

Brealey, R.A. et S.C. MYers (1991), Principles of Corporate Finance, New York, McGraw-Hill.

BROPHY, D.J. et J.M. SHULMAN (1992), «A finance perspective on entrepreneurship research », Entrepreneurship Theory and Practice, vol. 16, n 3, p. 61-71.

BROPHY, D.J. et J.M. ShULMAN (1993), «Financial factors which stimulate innovation », Entrepreneurship Theory and Practice, vol. 17, n 2, p. 61-75.

BYGRAVE, W.D. et C.W. HofER (1991), "Theorizing about entrepreneurship », Entrepreneurship Theory and Practice, vol. 15, $\mathrm{n}^{\circ} 2$, p. 13-22.

BYGRAVE, W.D. (1993), «Theory building in the entrepreneurship paradigm », Journal of Business Venturing, vol. 8, $\mathrm{n}^{\circ}$ 3, p. 255-280.

CHAN, K.C. et N.F. CHEN (1991), « Structural and return characteristics of small and large firms », Journal of Finance, vol. 46, n 4, p. 1467-1484.

DE VROEy, M. (1987), «La possibilité d'une économie décentralisée-Esquisse d'une alternative à la théorie de l'équilibre général », Revue Économique, p. 773-805.

DuKES, W.P., O.D. Bowlin et C.K. MA (1992), «Valuation techniques for closelyheld firms », Communication présentée au Fourth Annual International Research Symposium on Small Firm Finance, Waco, Texas.

Foss, N.J. (1993), « Theories of the firm: contractual and competence perspectives », Journal of Evolutionary Economics, vol. 3, p. 127-144.

Freear, J. et W.E. WeTzel (1992), «The informal venture capital market in the 1990s », dans D.L. Sexton et J.D. Kasarda (éd.), The State of the Art of Entrepreneurship, The Coleman Foundation, PWS-Kent, p. 462-486. 
GARTNER, W.B. (1988), "Who is an entrepreneur?" is the wrong question", American Journal of Small Business, vol. 12, $\mathrm{n}^{\circ} 4$, p. 11-32, republié dans Entrepreneurship Theory and Practice, été, 1989, p. 47-68.

GIBB, A.A. (1992), « Can academe achieve quality in small firms policy research?», Entrepreneurship and Regional Development, vol. 4, p. 127-144.

GiBSON, B. (1992), «The alternative to assuming "rationale" use of financial information within small firms », Communication présentée au Fourth Annual International Research Symposium on Small Firm Finance, Waco, Texas.

HALL, J. et C.W. Hofer (1993), «Venture capitalists' decision criteria in new venture evaluation », Journal of Business Venturing, vol. 8, $\mathrm{n}^{0} 1$, p. 25-42.

IVANCEVICH, J.M. (1991), «A traditional faculty member's perspective on entrepreneurship », Journal of Business Venturing, vol. 6, $\mathrm{n}^{\circ}$ 1, p. 1-7.

JEGADEESH, N. (1992), «Does market risk really explain the size effect? », Journal of Financial and Quantitative Analysis, vol. 27, n ${ }^{0} 3$, p. 337-351.

JENSEN, M.C. et W.H. MECKLING (1976), «Theory of the firm : managerial behavior, agency costs and ownership structure », Journal of Financial Economics, vol. $3, n^{\circ} 2$, p. $305-360$.

KAISH, S. et B. GILAD (1991), « Characteristics of opportunities search of entrepreneurs versus executives: sources, interests, general alertness », Journal of Business Venturing, vol. 6, $\mathrm{n}^{0} 1$, p. 45-61.

KeELEY, R.H. et L.A. TURKI (1992), «Risk-Return profiles of new ventures : an empirical study », Communication présentée au Fourth Annual International Research Symposium on Small Firm Finance, Waco, Texas.

KLINGE, B.F. (1992), «Causality between investment activity and cash flow of U.S. based non-financial firms », Communication présentée au Fourth Annual International Research Symposium on Small Firm Finance, Waco, Texas.

LANDSTRÖM, H. (1992), «The relationship between private investors and small firms : an agency theory approach », Entrepreneurship and Regional Development, vol. 4, no 3, p. 199-223.

MACMILlan, I.C. et J.A. KATZ (1992), «Idiosyncratic milieus of entrepreneurial research : the need for comprehensive theories ", Journal of Business Venturing, vol. $7, \mathrm{n}^{\circ} 1$, p. 1-8.

MCMAHON, R.G.P. (1990), «Expert systems and financial decision support in small businesses », International Small Business Journal, vol. 8, $\mathrm{n}^{\circ}$ 2, p. 23-33.

MCMahon, R.G.P., S. Holmes, P.J. Hutchinson et D.M. Forsaith (1993), Small Enterprise Financial Management: Theory and Practice, New York, Harcourt Brace Jovanovich. 
Modigliani, F. et M.H. Miller (1958), «The cost of capital, corporation finance, and the theory of investment », American Economic Review, vol. 48, n० 3, p. 261-297.

Monahan, Th.F., D.E. Stout et L.C. Barenbaum (1992), «Using small firm samples to value closely-held companies: an empirical analysis», Communication présentée au Fourth Annual International Research Symposium on Small Firm Finance, Waco, Texas.

MYERS, S.C. (1990), «Still searching for optimal capital structure», HEC International Conference.

NORTON, E. (1991), «Capital structure and small growth firms », Journal of Small Business Finance, vol. 1, n ${ }^{\circ}$ 2, p. 161-177.

Perryman, M.R. (1982), "Commentary on research in the field of entrepreneurship », dans C.A. Kent, D.L. Sexton et K.H. Vesper (éd.), Encyclopedia of Entrepreneurship, New York, Prentice-Hall, p. 377-379.

PETTIT, R.R. et R.F. SINGER (1985), «Small business finance : a research agenda », Financial Management, vol. 14, no 3, p. 47-60.

PetTy, J.W. (1991), «Research in small-firm entrepreneurial finance: a note on developing a paradigm », Journal of Small Business Finance, vol. 1, $\mathrm{n}^{\circ} 1$, p. 89-90.

Rosman, A.J. et H.M. O'NEILl (1993), «Comparing the information acquisition strategies of venture capital and commercial lenders: a computer-based experiment », Journal of Business Venturing, vol. 8, $\mathrm{n}^{0}$ 5, p. 443-460.

RuHNKA, J.C. et J.E. Young (1991), « Some hypotheses about risk in venture capital investing », Journal of Business Venturing, vol. 6, $\mathrm{n}^{\circ} 1$, p. 115-134.

SCHWERT, G.W. (1983), «Size and stock returns, and other empirical regularities », Journal of Financial Economics, vol. 12, n 1, p. 3-12.

SEXTON, D.L. (1988), « To grow or not to grow : It is your decision », Reflections on the Future, A National Symposium on Women in Entrepreneurship, BaldwinWallace College, Berea, Ohio.

SEXTON, D.L. et J.D. KASARDA (éd.) (1992), The State of the Art of Entrepreneurship, The Coleman Foundation, PWS-Kent.

SHARPE, W.F. (1964), «Capital asset prices : a theory of market equilibrium under conditions of risk », Journal of Finance, vol. 19, n 3, p. 425-442.

Shulman, J.M., R. CoOPER et D.J. Brophy (1992), «Capital structure life cycle: static process or dynamic evolution », Communication présentée au Fourth Annual International Research Symposium on Small Firm Finance, Waco, Texas.

Suret, J.M. et J.M. GAGNON (1990), « Coûts d'agence, fiscalité et PME: aspects théoriques et tests empiriques », Revue PMO, vol. 5, nº 2, p. 28-38. 